\title{
Whole Genome Sequence Resource for Fusarium oxysporum f. sp. capsici 14003, the Causative Agent of Pepper Wilt
}

\author{
Yongxin Shu, Mei Luo, ${ }^{\dagger}$ and Zhangyong Dong ${ }^{\dagger}$ \\ Innovative Institute for Plant Health, Zhongkai University of Agriculture and Engineering, Guangzhou \\ 510225, China
}

\begin{abstract}
Pepper wilt, caused by the fungal pathogen Fusarium oxysporum f. sp. capsici, is one of the main diseases on pepper. In this study, we obtained the whole genome sequence of the highly virulent $F$. oxysporum f. sp. capsici strain 14003 sequenced using the Illumina HiSeq 2000 platform. In total, 3.87 million paired-end reads were obtained and assembled into 796 scaffolds with a genome-wide length of $47.6 \mathrm{Mb}$. In addition, we filtered genes that may be related to specific virulence factors and performed a comparative analysis between the genome of strain 14003 and the sequenced genomes of 36 isolates. The genomesequencing results for strain 14003 will enhance the theoretical basis for pepper wilt prevention and control.
\end{abstract}

\section{Genome Announcement}

Pepper is an important vegetable and spice crop belonging to the nightshade family Solanaceae, which is cultivated in approximately 130 countries. The plant is a rich source of vitamin $C$ - and vitamin A-containing $\beta$-carotenoids, which are potent antioxidants (Serra et al. 2002; USDA-NASS 2003). Pepper wilt is the main disease on pepper, which is caused by Fusarium oxysporumf. sp. capsici, a highly virulent fungal pathogen of peppers (Rivelli 1989). Generally, the characteristic symptoms of Fusarium wilt of pepper include wilting, stunting, leaf chlorosis, slight vein clearing on the outer leaflets, vascular necrosis, and, ultimately, death (Altinok et al. 2019); and, under damp conditions, white mold may grow on the diseased parts of the plant. Fusarium wilt readily establishes and can spread rapidly in warm humid, environments and when plants are grown under monoculture conditions (Velarde-Félix et al. 2018). Chemical and biological control of pepper wilt are of limited efficacy, and there are currently no pepper varieties showing durable resistance to this disease (Mao and Mo 2019). Consequently, at present, there are no completely effective measures available for the control of pepper wilt. From a long-term control perspective, for the effective management of Fusarium wilt, it will be essential to examine the pathogenic mechanisms of $F$. oxysporum f. sp. capsici and to identify the associated virulence genes and their functions. Currently, however, there is a conspicuous lack of information relating to the genomic features and mechanisms of interaction underlying the pathogenicity of strain 14003 against pepper. Therefore, sequencing and analyzing the genome of this pathogen has become an urgent prerequisite.

In this study, we determined the genome sequence of $F$. oxysporum f. sp. capsici strain 14003, which was isolated from Guangdong Province, China. F. oxysporum f. sp. capsici 14003 was cultured for 5 days on potato dextrose medium at $25^{\circ} \mathrm{C}$. Genomic DNA was

\footnotetext{
†Corresponding authors: Z. Dong; dongzhangyong@zhku.edu.cn; and M. Luo; 08luomei@163.com
}

The author(s) declare no conflict of interest.

Accepted for publication 23 November 2020.

\section{Funding}

This work was funded by the Key projects of Guangdong Universities (Natural Science) (grant number 2019KZDXM040), and the National Natural Science Foundation of China (grant number 31301627).

\section{Keywords}

Fusarium oxysporum f. sp. capsici, pepper wilt, whole genome sequence 
Table 1. Information on the sequenced genomes of 37 Fusarium isolates

\begin{tabular}{|c|c|c|}
\hline Name & Assembly & Total sequence length \\
\hline Fusarium beomiforme & GCA_002980475.2 & $46,521,357$ \\
\hline F. fujikuroi IMI 58289 & GCA_900079805.1 & $43,832,314$ \\
\hline F. mangiferae & GCA_900044065.1 & $46,292,722$ \\
\hline F. verticillioides 7600 & GCA_000149555.1 & $41,844,914$ \\
\hline F. coffeatum & GCA_003316985.1 & $37,769,732$ \\
\hline F. oxysporum f. sp. cubense race 1 & GCA_000350365.1 & $52,926,277$ \\
\hline F. oxysporum f. sp. cubense tropical race 454006 & GCA_000350345.1 & $47,657,417$ \\
\hline F. oxysporum f. sp. cubense race 4 & GCA_000260195.2 & $46,553,780$ \\
\hline F. oxysporum f. sp. cubense & GCA_007994515.1 & $48,588,396$ \\
\hline F. oxysporum f. sp. lycopersici 4287 & GCA_000149955.2 & $61,386,934$ \\
\hline F. oxysporum f. sp. conglutinans race 254008 & GCA_000260215.2 & $53,575,352$ \\
\hline F. oxysporum f. sp. cepae & GCA_003615085.1 & $53,425,788$ \\
\hline F. oxysporum f. sp. cepae & GCA_003615095.1 & $51,407,732$ \\
\hline F. oxysporum f. sp. cepae & GCA_003615075.1 & $51,009,701$ \\
\hline F. oxysporum f. sp. lycopersici MN25 & GCA_000259975.2 & $48,637,398$ \\
\hline F. oxysporum f. sp. radicis-lycopersici 26381 & GCA_000260155.3 & $49,359,289$ \\
\hline F. oxysporum f. sp. raphani 54005 & GCA_000260235.2 & $53,499,362$ \\
\hline F. oxysporum f. sp. radicis-cucumerinum & GCA_001702695.2 & $52,908,293$ \\
\hline F. oxysporum f. sp. vasinfectum 25433 & GCA_000260175.2 & $52,914,414$ \\
\hline F. oxysporum f. sp. narcissi & GCA_004141715.1 & $57,517,562$ \\
\hline F. oxysporum f. sp. pisi HDV247 & GCA_000260075.2 & $55,188,216$ \\
\hline F. oxysporum f. sp. melonis 26406 & GCA_000260495.2 & $54,034,280$ \\
\hline F. oxysporum & GCA_003615165.1 & $50,515,499$ \\
\hline F. oxysporum & GCA_003615155.1 & $53,038,185$ \\
\hline F. oxysporum & GCA_003615185.1 & $50,292,220$ \\
\hline F. oxysporum Fo47 & GCA_000271705.2 & $49,664,628$ \\
\hline F. oxysporum NRRL 32931 & GCA_000271745.2 & $47,906,303$ \\
\hline F. oxysporum Fo5176 & GCA_000222805.1 & $54,767,602$ \\
\hline F. oxysporum & GCA_900096695.1 & $49,085,590$ \\
\hline F. oxysporum & GCA_003615115.1 & $54,787,818$ \\
\hline F. langsethiae & GCA_001292635.1 & $37,543,021$ \\
\hline F. poae & GCA_001675295.1 & $46,476,831$ \\
\hline F. graminearum $\mathrm{PH}-1$ & GCA_000240135.3 & $36,458,046$ \\
\hline F. pseudograminearum CS3096 & GCA_000303195.2 & $36,973,259$ \\
\hline F. sporotrichioides & GCA_003012315.1 & $37,429,079$ \\
\hline F. venenatum & GCA_900007375.1 & $38,660,186$ \\
\hline F. oxysporum f. sp. capsici & GCA_014770115.1 & $47,555,656$ \\
\hline
\end{tabular}

extracted from fresh mycelium using the cetyltrimethylammonium bromide method (Kim et al. 2010). DNA sequencing was carried out using an Illumina HiSeq 2000 Sequencer operating in 100-bp paired-end mode, with library insert sizes of $300 \mathrm{bp}$. In total, 3.87 million raw reads were generated, from which low-quality data were filtered using fastqc and Trimmomatic. After quality filtering, high-quality reads were de novo assembled using Abyss, Velvet, and SOAPdenovo. In total, we constructed 796 scaffolds for a genome size of 47,555,656 bp, with an $\mathrm{N}_{50}$ value of $209,562 \mathrm{bp}$. The overall $\mathrm{G}+\mathrm{C}$ content of the strain 14003 assembly was $51.0 \%$ and the genome coverage was $59.0 \times$. The total length of strain 14003 is within the size range of previously published Fusarium genomes (Table 1). BUSCO (Simão et al. 2015) analysis showed that the gene models predicted that the three genomes covered $99 \%$ of the single-copy conserved fungal genes, indicating the near-completeness of the assemblies. We used AUGUSTUS v3.3.3 (Stanke et al. 2004) to annotate the genome, and 15,762 protein-encoding genes were predicted, with an average length of 510 amino acids.

Genes were then functionally assigned using BLAST (version 2. 7. 1+) (Kent 2002) against the following databases: SwissProt, NR, KOG, KEGG, MEROPS, DFVF, LIPABASE, Cytochrome P450, PHI Database, and CAZymes, for E-values $\leq 1 \mathrm{e}^{-5}$. Additionally, within the F. oxysporum f. sp. capsici 14003 genome, we identified potential pathogenicity and virulence-related genes, including 3,389, 863, and 6,187 genes encoding P450 enzymes, CAZymes, and PHI proteins, respectively.

We predicted 15,762 protein-encoding genes in the F. oxysporum f. sp. capsici 14003 genome, which is considered a moderate number. Gene families among 37 other sequenced genomes of Fusarium isolates were classified based on orthologous clustering using the 
Orthofinder platform (Emms and Kelly 2015), and yielded 1,615 single-copy orthologs. The whole genomes of the type isolates representing the other Fusarium isolates have previously been sequenced and are available in the NCBI database (Table 1).

We compared the 37 Fusarium genomes and identified 232 genes specific to strain 14003. SignalP (version 4.1), TMHMM V2.0 TargetP (V1.1), and ProtComp predicted that 23 of these 232 specific genes are secreted proteins. We used the $\mathrm{PHI}$ database to annotate these 232 proteins and, among these, the deletion of 90 proteins has been shown to result in reduced virulence. Following deletion, the pathogenicity 10 proteins, classified as effectors, was completely lost, and we accordingly speculate that these might be pathogenic factors. In total, 7 proteins have been shown to increase virulence, whereas 77 proteins were annotated as unaffected, and a further 6 proteins were classified as lethal. The other 23 proteins were annotated as mixed results. We subsequently used PFAM (Finn et al. 2008) to predict the domains of the 232 specific proteins. In total, we annotated 104 genes in the F. oxysporum $\mathrm{f}$. sp. capsici 14003 genome, among which 35 genes collectively belonged to the polysaccharide lyases (EC 4.2.2.-) (PLs) based on their CAZymes identifications. PLs play significant roles in breaching plant cell wall defenses after degrading cellulose and pectin and, among the 37 Fusarium genomes we assessed, that of strain 14003 was found to have the highest number of PL genes. PL1 and PL3 are the most common pectin lyases found in plant pathogens and, in the present study, we detected 14 and 11 copies of PL1 and PL3, respectively, in F. oxysporum capsici 14003. Among six other PL members (PL11, PL20, PL22, PL4, PL7, and PL9), it has previously been hypothesized that PL9, PL 20, and PL22 are specific to F. virguliforme and Nectria hematococca (teleomorph: F. solani), and are not found in other plant fungal pathogens or oomycetes (Fei et al. 2019). However, we detected both PL9 and PL22 domains in the strain 14003 genome. In other Fusarium strains, PL11 and PL4 have been observed. Notably, we identified a novel $\beta$-elimination (PL7) in the strain 14003 genome. To analyze the secondary metabolite synthesis potential of strain 14003, we used antiSMASH 3.0 (fungal version) (Weber et al. 2015) to identify secondary metabolite biosynthesis gene clusters in the genome of strain 14003, and performed similar analyses for the other 36 Fusarium genomes for comparative purposes. We accordingly identified a total of 44 key biosynthetic genes in the strain 14003 genome that are potentially associated with secondary metabolites, among which there are 21 genes encoding nonribosomal peptide synthetases (NRPS), 16 encoding polyketide synthases (PKS), 5 genes encoding PKSNRPS, and 1 gene encoding terpene synthase. The remainder included single biosynthesis genes for other secondary metabolites. On the basis of the comparison of the secondary metabolite-associated genes identified in the 37 Fusarium genomes, it is clear that the number of genes annotated as NRPS and PKS in the F. oxysporum f. sp. capsici 14003 genome is considerably higher than that in most Fusarium strains, although similar to that in the F. beomiforme genome. In contrast, we detected fewer PKS-NRPS and other secondary metabolite-associated genes in the $F$. oxysporum f. sp. capsici 14003 genome than in the other 36 Fusarium genomes.

Data availability. The whole genome data presented in this paper have been deposited in GenBank under the accession number JACYOB000000000 (BioProject PRJNA664524).

\section{Literature Cited}

Altinok, H. H., Yüksel, G., and Altinok, M. A. 2019. Pathogenicity and phylogenetic analysis of Fusarium oxysporum f. sp. capsici isolates from pepper in Turkey. Can. J Plant Pathol. 42:279-291.

Emms, D. M., and Kelly, S. 2015. OrthoFinder: Solving fundamental biases in whole genome comparisons dramatically improves orthogroup inference accuracy. Genome Biol. 16:157.

Fei, S., Czislowski, E., Fletcher, S., Peters, J., Batley, J., Aitken, E., and Mitter, N. 2019. Small RNA profiling of Cavendish banana roots inoculated with Fusarium oxysporum f. sp. cubense race 1 and tropical race 4. Phytopathol Res. 1:22.

Finn, R. D., Tate, J., Mistry, J., Coggill, P. C., Sammut, S. J., Hotz, H. R., Ceric, G., Forslund, K., Eddy, S. R., Sonnhammer, E. L., and Bateman, A. 2008. The Pfam protein families database. Nucleic Acids Res. 36:D281-D288.

Kent, W. J. 2002. BLAT-The BLAST-Like alignment tool. Genome Res. 12:656-664.
Kim, J. S., Seo, S. G., and Jun, B. K. 2010. Simple and reliable DNA extraction method for the dark pigmented fungus, Cercospora sojina. Plant Pathol. J. 26: 289-292.

Mao, T. T., and Mo, W. D. 2019. Advances in prevention and control of pepper wilt. Chin. J. Guizhou Agric. Sci. 47:49-53.

Rivelli, V. C. 1989. A wilt of pepper incited by Fusarium oxysporum f. sp. capsici formae specialis nova. Louisiana State University, Baton Rouge, LA, U.S.A.

Serra, I., Yamamoto, M., Calvo, A., Cavada, G., Báez, S., Endoh, K., Watanabe, H., and Tajima, K. 2002. Association of chili pepper consumption, low socioeconomic status and longstanding gallstones with gallbladder cancer in a Chilean population. Int. J. Cancer 102:407-411.

Simão, F. A., Waterhouse, R. M., loannidis, P., Kriventseva, E. V., and Zdobnov, E. M. 2015. BUSCO: Assessing genome assembly and annotation completeness with single-copy orthologs. Bioinformatics 31:3210-3212. 
Stanke, M., Steinkamp, R., Waack, S., and Morgenstern, B. 2004. AUGUSTUS: A web server for gene finding in eukaryotes. Nucleic Acids Res. 32:W309W312.

USDA-NASS. 2003. Vegetables: 2002 Summary. United States Department of Agriculture-National Agricultural Statistics Service. Vg 1-2. https://downloads.usda.library.cornell.edu/usda-esmis/files/02870v86p/qj72p986q/Or967625k VegeSumm-01-29-2003.pdf
Velarde-Félix, S., Garzón-Tiznado, J. A., Hernández-Verdugo, S., López-Orona, C. A., and Retes-Manjarrez, J. E. 2018. Occurrence of Fusarium oxysporum causing wilt on pepper in Mexico. Can. J. Plant Pathol. 40:238-247.

Weber, T., Blin, K., Duddela, S., Krug, D., Kim, H. U., Bruccoleri, R., Lee, S. Y., Fischbach, M. A., Müller, R., Wohlleben, W., Breitling, R., Takano, E., and Medema, M. H. 2015. AntiSMASH 3.0-A comprehensive resource for the genome mining of biosynthetic gene clusters. Nucleic Acids Res. 43:W237-W243. 